\title{
Comparison of Spraying and Nebulized Lidocaine in Patients undergoing Esophago-Gastro-Duodenoscopy
}

\author{
Papiroon Noitasaeng*, Phongthara Vichitvejpaisal ${ }^{*}$,Uayporn Kaosombatwattana **, Tassanee Jaiyen*, \\ Suwannee Siriwongsa***
}

Co-Authors: Professor Phongthara Vichitvejpaisal MD, PhD*.

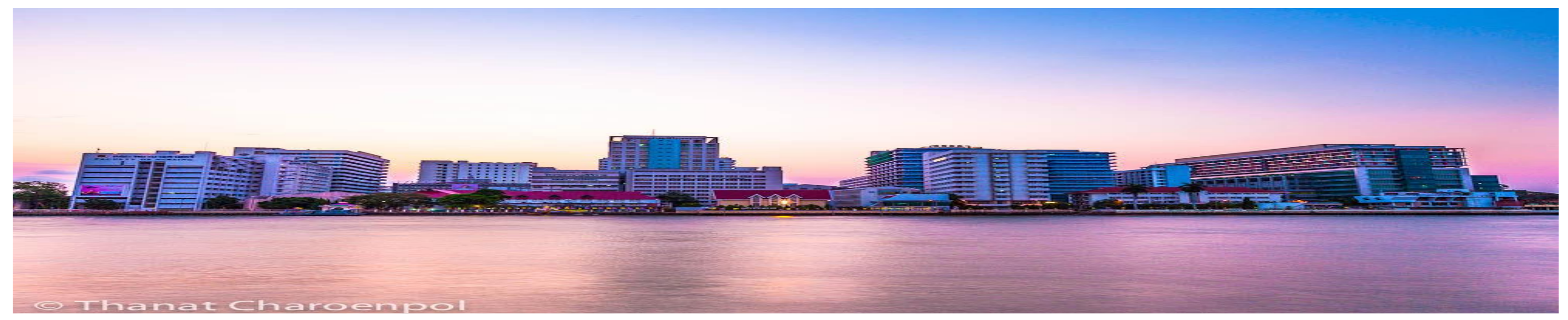

Background and goal of study : Esophago-gastroduodenoscopy (EGD) was performed under the topical anaesthesia of the pharynx with either $2 \%$ viscous or $10 \%$ liquid lidocaine because it yields a rapid onset with a high safety margin. However, spraying lidocaine was found to be an annoying maneuver to patients, while nebulized lidocaine appeared to efficiently suppress gags and cough reflexes in airway anaesthesia. In this paper, we compare the effectiveness of spraying and nebulized lidocaine for patients undergoing EGD.

Material and Methods: This study has been approved by Siriraj Institutional Review Board, and a written informed consent was obtained from all subjects. Included were a total of 110 patients undergoing elective EGD, with a history of neither lidocaine intolerance nor irritable airways due to smoking, COPD, upper respiratory infection, asthma, cardiac and pulmonary diseases and allergy to lidocaine. All patients were randomized into two groups: A - where 5 puffs (10mg/puff) of spraying lidocaine were administered four times at 5-minute intervals, up to a total dose of $200 \mathrm{mg}$; and B where $250 \mathrm{mg}$ of nebulized lidocaine was administered via a nebulization kit with an oxygen face mask of 7 LPM for 15 minutes prior to the commencement of EGD. The procedure was performed by the same board-certified endoscopist. The co-researcher who was blinded to the lidocaine administration technique assessed the ease of esophageal instrumentation as either difficult, poor, fair or excellent. Both the endoscopist and the patients expressed their satisfaction by using the Numerical Rating Scale. Data were expressed as mean and standard deviation. $\mathrm{P}<0.05$ with a $95 \%$ confidence interval was considered statistically significant difference.

* Department of Anesthesiology

** Department of Medicine

*** Department of Perioperative Nursing Faculty of Medicine Siriraj Hospital, Mahidol University, Bangkok 10700, THAILAND
Results and Discussion: Demographic data were not significant differences between the groups. One patient in group A was dropped out due to the extended protocol, and other two in group B due to the incidence of bronchospasm and recall of upper respiratory tract infection. The endoscopist expressed her satisfaction with instrumentation, which showed significant difference between group $A$ and group $B$ as $84.8 \pm 8.3$ and $79.2 \pm 11.2$ respectively. The co-researcher also found that group A patients responded to the ease of esophageal instrumentation better than those in group B. This might due to up to $60 \%$ of lidocaine was lost to the atmosphere or in patients' mouth during the nebulization and it was not easy to figure out the dosage of drug to alleviate the discomfort during instrumentation. However, nebulized lidocaine had significant advantages over spraying lidocaine, with better acceptance in patients undergoing EGD

Table . The endoscopist and the co-researcher assessed the procedural effectiveness under the numerical rating scale (mean \pm SD) and number of patients' response $n(\%)$ in consequence.

\begin{tabular}{lcccc}
\hline & $\begin{array}{c}\text { Patients' } \\
\text { response }\end{array}$ & $\begin{array}{c}\text { Group A } \\
(\mathbf{n = 5 4})\end{array}$ & $\begin{array}{c}\text { Group B } \\
(\mathbf{n = 5 3})\end{array}$ & p-value \\
\hline Endoscopist & & $84.8 \pm 8.3$ & $79.2 \pm 11.2$ & 0.004 \\
Co-researcher & difficult & $0(0)$ & $0(0)$ & \\
& poor & $1(1.9)$ & $4(7.5)$ & \\
& fair & $27(50)$ & $40(75.5)$ & \\
& excellent & $26(48.1)$ & $9(17.0)$ & \\
\hline
\end{tabular}

Conclusion: The endoscopist expressed her approval of spraying lidocaine for taking less time to start the procedure, ease for instrumentation, less gag reflex during the procedure, less presence of hypersecretion, and smooth operation. However, participants favored nebulized lidocaine administration.

Keywords: anesthetic technique-topical, esophago-gastrouodenoscopy. 